\title{
BMJ Open Quality Patients at the centre of care: debriefing patients after caesarean section
}

\author{
Claire Dougan, Emma Smith, Jennifer Ploski, Arthur Mc Nally, Katie Johnston
}

To cite: Dougan C, Smith E, Ploski J, et al. Patients at the centre of care: debriefing patients after caesarean section. BMJ Open Quality 2019;8:e000454. doi:10.1136/ bmjoq-2018-000454

- Additional material is published online only. To view please visit the journal online (http://dx.doi.org/10.1136/ bmjoq-2018-000454)

Previously presented at Society of Maternal Fetal Medicine conference 2018 (poster/ abstract).

Received 18 June 2018 Revised 23 August 2019 Accepted 6 October 2019
Check for updates

(c) Author(s) (or their employer(s)) 2019. Re-use permitted under CC BY-NC. No commercial re-use. See rights and permissions. Published by BMJ.

Obstetric Department, Royal Jubilee Maternity Service, Belfast, UK

Correspondence to

Dr Claire Dougan;

cdougan06@qub.ac.uk

\section{ABSTRACT}

Empowering patients and working collaboratively to improve healthcare is a focus for current healthcare development strategies. In obstetric practice, working in collaboration with women to discuss mode of delivery after caesarean section has been shown to impact women's choice. A preliminary survey in our tertiary maternity unit showed $20 \%$ of women gained 'full clarity' with the information they received after caesarean-section regarding indication, complications and impact on future delivery. Focus groups identified what information patients required about their surgery. General practitioners (GP) highlighted a lack of communication between secondary and primary care. Junior obstetricians surveyed felt uncertain about debriefing. The aim was to improve monthly 'full clarity' scores to $30 \%$ in 6 months. Our team introduced a debrief form, filled by the operator and kept in maternity notes. The operator verbally debriefed as part of their current standard practice. Junior doctors postoperatively debriefed the patient using the form. The triplicate form ensured both patients and primary care received a copy. It was anticipated that in the long term, this would provide consistent information and aid informed decision making in subsequent pregnancies. Review of the forms' use was undertaken weekly. Multidisciplinary input identified barriers to uptake. Plan, Do, Study, Act cycles promoted the project, disseminated results, evidenced growing participation and rewarded consistent form use. Patient groups critiqued the form layout. Teaching aimed to improve junior doctor confidence in debriefing discussions. Sustained participation resulted in effective form uptake. Patient 'full clarity' scores improved to $60 \%$ with minimal impact on workload. Teaching successfully improved junior doctor confidence as evidenced by increased documented debrief discussions. GP focus groups felt empowered to counsel women consistently about their surgery increasing clarity for everyone. This project highlights that patient satisfaction improves when we listen to their views and commit to change.

\section{PROBLEM}

Communication is acknowledged by the General Medical Council as a principal domain in the duties of a doctor. ${ }^{1}$ The National Health Service highlights that improved communication with patients increases satisfaction with care and leads to improved outcomes. ${ }^{2}$

Communication is central in obstetric practice when discussing mode of delivery after caesarean-section. There is a continued global drive to decrease caesarean-section rates and promote vaginal birth after caesarean-section (VBAC). ${ }^{3}$ Dedicated antenatal clinics, supporting women through the informed decision-making process on mode of birth after a primary caesarean delivery, has been found to improve VBAC attempt rates. ${ }^{45}$

The overarching aim of this work-stream was to review why patients chose their intended mode of delivery in the Royal Jubilee Maternity Hospital (RJMH) Belfast. The National Health Service recognises that involving patients often improves efficiency in design and joining up services, in this case, between initial and subsequent gestations. ${ }^{6}$

RJMH, is a tertiary maternity unit with 5500 deliveries annually. Elective caesarean sections are undertaken in the elective theatre suite while emergency caesarean sections occur on delivery suite. Postoperatively, all share the same postnatal wards, remain as inpatients on average for 2 days and are discharged with community midwife involvement. General practitioners (GP) review at 6weeks post partum. Standard practice debriefing after caesarean section occurred verbally, shared between the operator and the junior medical staff on the postoperative ward. RJMH 'Birth Choices' antenatal clinic is where women with previous caesarean-sections receive their antenatal care in subsequent pregnancies. A multidisciplinary team of obstetricians and specialist midwives discuss the options of VBAC and elective repeat caesarean-section (ERCS).

Anecdotal evidence and staff perception suggested patients who returned in a subsequent pregnancy had little knowledge about the indication for their surgery or their suitability for VBAC. This lead to a lengthy antenatal process discussing previous events and the option of VBAC when the patient had already formulated their perception of events and intended mode of delivery. Patients frequently could not recall postoperative discussions. Some cited different information given by different care providers, causing patient distress. The project team felt that this hindered women in making informed 
decisions and that discussion in subsequent pregnancy was information given 'too late'. The team believed improving communication at the time of initial caesarean-section would be beneficial. Different care providers giving conflicting information to women, highlighted poor inter-professional communication regarding VBAC and its safety profile.

Our baseline survey, designed from patient identified themes, reported that $20 \%$ of women gained 'clarity' with the information provided to them about their operation. Patients also identified GPs as the most common healthcare provider to seek advice from regarding suitability for VBAC. The project intended to create a robust way in which to measure and improve patients' clarity regarding the information they were given.

We aimed to improve 'full clarity' from $20 \%$ to $30 \%$ within a 6-month project time period. This modest figure was chosen as a patient's perception of 'clarity' may not be achievable in the immediate postnatal period due to competing demands on their attention. We introduced small changes that would not impact on workload and had a short time frame to implement these. The project aimed to provide consistent information to GPs.

\section{BACKGROUND}

Factors influencing women's decision on mode of birth are multifactorial. While the health of both mother and baby is reported as a major determinant in deciding mode of delivery, natural experience, recovery time, avoidance of pain and convenience of timing a planned birth were other factors reported in the literature. ${ }^{7}$ Medical advice was one of the biggest influences on women's choice shown in a recent Swiss study. ${ }^{7}$ The issue of 'safety' was a predictor to decrease the probability of choosing VBAC. ${ }^{7}$ While both VBAC and ERCS have low maternal and neonatal risks, ${ }^{8}$ information on safety given by care providers may emphasise elective caesarean-section as a 'safe' option. ${ }^{7}$ Fenwick et al concur that medical advice emphasising the safety of caesarean-section was associated with women's decision for a repeat caesarean-section. ${ }^{9}$

Women have identified that they often receive contrasting information about mode of birth options. ${ }^{10}$ A consistent confident attitude regarding VBAC by the healthcare provider is important to women. ${ }^{11}$ Clinicians in countries with high VBAC rates agree that strengthening women's trust that VBAC is a viable option, is an important aspect in why women choose VBAC. ${ }^{12}$ Early information about the initial caesarean-section, the reason why it occurred and suitability of VBAC is important to women. ${ }^{11}{ }^{13}$ Debriefing as to why the caesarean-section was required and information on VBAC was requested 'as soon as possible'. 'A Canadian study showed that women's decision-making process commonly began during the interpregnancy interval and would benefit from decision support as early as immediately following primary caesarean-section. In some cases, support was required before patients would contemplate a future pregnancy. ${ }^{13}$ In this

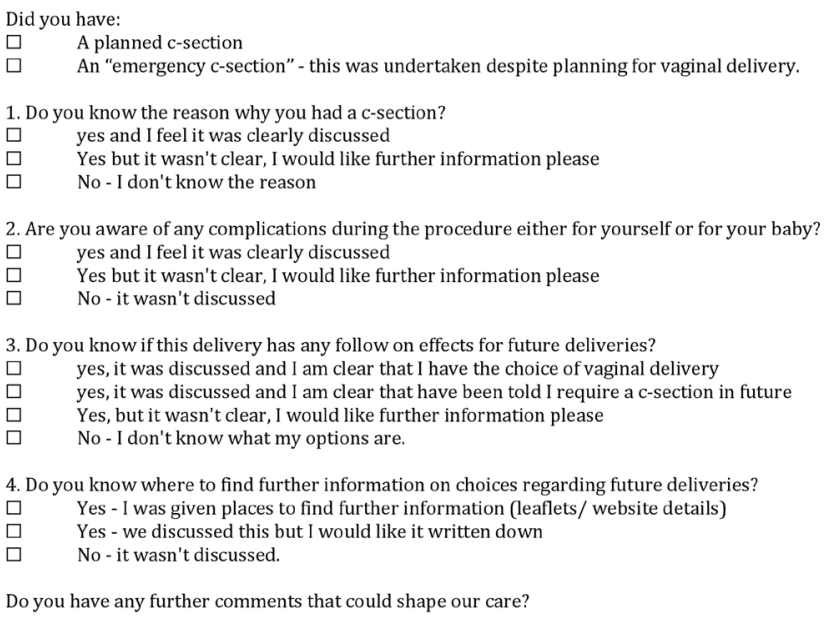

Do you have any further comments that could shape our care?

Figure 1 Patient clarity survey questions. Only 'clear' answers were counted as a positive response.

regard we may be failing some patients who, without adequate information given at the time of caesarean-section, may never embark on subsequent pregnancies and do not feature in our service again.

Verbal information was preferred, but leaflets were also important. In the absence of information from care providers about their first birth, participants relied on personal knowledge from their caesarean experience which risks inaccuracy, and information from peers and the internet which may not be specific to their clinical situation. ${ }^{13}$ The National Institute of Clinical Excellence (NICE) recommend both verbal and printed information be available to these women. ${ }^{14}$

Shared decision making and empowering the women to make their own choice was another strong theme in recent studies. ${ }^{1115}$

\section{BASELINE MEASUREMENT}

To confirm a need for change, a focus group of five women identified their information needs. While the team's aim was to impart information regarding VBAC, women highlighted they knew little about indication and complications of their surgery. Using the themes of indication, complications and impact on future delivery, we then surveyed 25 women at day 1-2 postcaesarean-section in December 2016 (figure 1). Women were randomly selected representing both elective and emergency caesarean-section. $20 \%$ of women achieved 'clarity' with the information in all four areas.

Preceding this project, debriefing after caesarean section did not consistently meet NICE guidance. It occurred verbally and sporadically, shared between the operator and junior medical staff on the postnatal ward. Consultant obstetricians and senior obstetric trainee operators, reported barriers to postoperative patient debriefing. They discussed that job requirements caused inconsistent availability to debrief women the following day. Often their debrief occurred immediately postoperatively in 
the recovery room. The operators believed that junior doctors also debriefed postnatally as part of their standard day 1 review. There was no documentation of these interactions. A focus group of six junior doctors expressed barriers to debriefing included time restraints to review detailed intra partum notes and lack of confidence in what to discuss. If suitability for VBAC was not written in the operative note, junior doctors would not broach this. Women surveyed felt discussion immediately after delivery was suboptimal, as they were unable to recount the information. Hunger, emotion, tiredness and the attention the new baby required were cited as reasons for this. They proposed patient specific written information be available so that they could revisit these points at a date suitable to them.

When discussing suitability for VBAC, we asked women in the baseline survey who they would ask for advice regarding this. While some mentioned family members and obstetricians, 13/25 reported asking their GP and others were unsure who to ask. A focus group of seven GP's within the Belfast catchment area responded to our survey. Their responses showed that while they were infrequently asked to clarify information regarding obstetric surgery, they would not feel confident in discussing these issues and had poor access to information from secondary care. An example debrief template met with positive feedback with all agreeing that it would be beneficial in their job. We invited the community midwifery team to participate but failed to achieve a response within our 6-month period.

The project focused on the introduction of patient specific written information to assist both patient and GP in the debriefing process and teaching for junior doctors on debriefing. Data collection occurred weekly and included type of caesarean section, grade of operator, documented debrief by operator, debrief form completed and documented debrief by junior doctor. Our outcome measure was the patient clarity score using the original survey questions. We evaluated if improvement was secondary to the written information by reviewing form uptake with weekly analysis. We also reviewed evidence of debriefing in the notes by junior doctors on a monthly basis.

\section{DESIGN}

The improvement team involved a lead senior obstetric trainee, two consultant supervisors, two junior obstetric trainees and midwifery team leaders in each clinical area involved in the care of patients with caesarean-sections in RJMH. Involvement of the 'Specialist Trainee Engaged in leadership Programme' within the Belfast Trust gave added multidisciplinary experience and guidance to the quality improvement process. The project was registered with the local quality improvement department and as such did not require ethical approval.

Baseline data was collated and discussed. A patient pathway identified all care providers that interacted with these patients. Barriers to clear consistent patient information were addressed. Appraisal of all suggested interventions included cost effectiveness, ease of implementation and patient benefit. The team agreed interventions which were beneficial and achievable. The project focused on; promotion of debriefing as a significant aspect of care, introduction of a debrief form and education of junior doctors in methods of debriefing. We assumed slow uptake of new documentation and focused a number of Plan Do Study Act (PDSA) cycles on project promotion and highlighting the benefits to physicians. Baseline results were presented at the department meeting to include the wider team in development of the project and promote its implementation. Patient experiences from the December survey were used to highlight the difficulties women have retaining verbal information and the importance of written information in empowering patients to understand events.

\section{Patient involvement}

Patients were not involved in setting the research question; however, two structured patient focus groups were involved in designing the clarity questionnaire and contributing to the evolution of the intervention. Patients rated the overall debriefing process throughout the project by way of the clarity questionnaire, monthly results of which formed the outcome measure. Their identification of primary care providers as an important resource for debriefing expanded our project to include GP's opinions on the intervention tool. Patients were not involved in agreeing plans for dissemination of results but will be informed through written communication in RJMH maternity wards and clinics.

\section{Measures}

Process measure: (1) weekly use of the debrief form; (2) monthly percentage documented debrief in maternity notes.

Outcome measure: monthly patient 'full clarity' rating. Balancing measure: time taken to fill form.

\section{STRATEGY}

PDSA cycles implemented interventions and measures identified their impact. As both elective and emergency cases were recovered in the same postnatal ward, interventions were implemented for both caesarean-section types to avoid confusion for postnatal staff. Weekly data collection and review captured 10 cases of adequate emergency and elective operation mix. Anonymised data included type of caesarean-section, grade of operator, documented debrief in clinical notes by operator, debrief form completed and documented debrief by junior doctor on day 1 postoperatively. These markers identified process measures, where implementation was successful and what operator groups required more project promotion. In patients who had the debrief form completed, a repeat clarity survey was undertaken. We chose this method because we wanted to identify whether implementation 
of the form changed clarity scores compared with baseline results. We also identified degree of successful form implementation. We reviewed debrief documentation in the patients notes on a monthly basis. We introduced junior doctor teaching and promotion of the importance of debriefing to affect change.

PDSA 1: aim: improve consistency of information. Intervention: introduction of debrief form. 4 February 2017 The first draft form had been printed in previous years but implementation had been problematic. We felt this was a cost-effective start. This form was based on doctor feedback that a simple form requiring minimal writing was required. It contained generic information discussing VBAC. An area discussing complications of the surgery was absent. We implemented this after presenting baseline findings at the department meeting in January 2017. New junior doctors joining the hospital service in February were informed of this practice. Emails reminded all staff about this project and its continuous review. Results showed 4/10 patients received the debrief form in the first week. The median clarity score was $3 / 4$ with one patient reporting 'full clarity'.

PDSA 2: aim: to improve uptake of debrief form. Intervention: 'message of the month' on the daily medical handover document.12 February 2017

Multidisciplinary typed handover on the delivery suite is doctor lead between the day and night staff at 08:00 and 20:00. A footer entitled 'message of the month' was introduced in February, which alerted staff that debriefing forms were available in all obstetric theatres and were a requirement for all caesarean-section patients. Debrief form use improved after this.

PDSA 3: aim: to improve uptake of debrief form. Intervention: feedback of results to elective and emergency theatre staff. 19 February 2017

Reviewing 2 weeks data, the team fed back to the elective and emergency theatre midwifery team leaders how well their area was embracing the debrief form. We discussed that uptake was dependent on the availability of the form at the end of the operative procedure. Forms were then included in the 'operative pack'. From this point onwards, we undertook monthly feedback to the theatre staff and promoted good practice in preference to punitive measures. Where patients mentioned staff specifically, we copied this to the staff member. This incentivised use of the form as a marker of good medical or midwifery practice. Midwives began reminding doctors to use the form and use increased.

PDSA 4: aim: to improve uptake of debrief form. Intervention: feedback of results to all doctors. 28 February 2017

Results were emailed to all obstetric staff. Use of the form was increasing and the median patient clarity score had increased from $3 / 4$ in December to 3.5/4 in February. The monthly 'full clarity' score increased to $30 \%$ of all patients surveyed. Promotion of these results showed the debriefing form as a successful intervention and increased 'buy in' from staff. Continued staff engagement empowered staff to feel proud that their work was making a difference.

PDSA 5: aim: to increase documented day 1 debriefing among junior doctors. Intervention: teaching junior doctors on debriefing. 5-20 March 2017

Junior doctors from the project team undertook semiformal teaching and discussion of the debrief process. This peer-to-peer teaching allowed a relaxed environment where the staff could voice their uncertainties. Where junior doctors often rotate through different hospital trusts, it was important to clarify that debriefing is a required role of the junior doctor rather than an optional aspect of care. It is used in conjunction with the operator debrief to ensure optimal patient understanding. This cycles success was measured by the increase in the number of debrief interactions documented in the maternity notes.

PDSA 6: aim: to improve uptake of debrief form. Intervention: 'cupcake challenge'. 20 March 2017

The obstetric staff in the elective operating theatre comprised of a consultant and junior trainee, while the emergency theatre operators were commonly middle grade and senior trainees. To increase compliance with the project, the 'Cupcake Challenge' encouraged friendly competition between these two clinical areas. The competition rewarded consistent use of the form over a 2-week period. This increased awareness of the project and proved to be the most successful intervention. Where previously the project had been understood by staff as to involve only doctors, this promotion encouraged midwives to get involved and ensure that all patients received this form before leaving the theatre/delivery suite area.

PDSA 7: aim: to improve uptake of debrief form. Intervention: monthly midwifery labour ward update. 9 April 2017

In April, elective theatre promotion of the form was encouraging, however, delivery suite staff awareness of the intervention was inconsistent. We realised that this was due to the large number of staff working various shift patterns including night shifts. Senior midwifery leaders included this on the monthly labour ward update, designed to reach all midwives over a 3-month period. This was undertaken sporadically and proved ineffectual to form uptake.

PDSA 8: aim: to improve documented operator debriefing. 'Animal league table'. 15 May 2017

Data showed variable staff engagement. The 'engagement lag' was pronounced on the delivery suite where patients had most to gain from careful debriefing after a labour process ending in emergency caesarean-section. To draw attention to this we designed an 'animal league 
table' displayed on the main notice board in the delivery suite staffroom. Each trainee doctor was assigned an animal to represent him/her. This encouraged discussion surrounding the project. People were privately informed which animal represented them. This allowed comparison of individual practice with peers without undermining. Documented verbal debriefs and debrief form use increased.

PDSA 9: aim: to improve patient satisfaction with debriefing process. Intervention: patient focus group critiquing debrief form

Throughout the project, the team received feedback from the patients being surveyed about the debrief process. Patients were mainly positive about the verbal debrief from the operator and the consistent message from the junior doctor. They also appreciated the written information to reiterate these points. A focus group of five patients critiqued the form throughout February. Suggestions to include an area discussing complications were reiterated. The form, designed by doctors to limit lengthy writing, they felt was cluttered. It listed numerous indications for caesarean-section, where only one would apply to the patient receiving it. They valued short sentences explaining personal reasons for caesarean-section. They suggested generic information on VBAC could be covered by other information sources. They preferred specific information about whether VBAC was applicable to them. They requested reputable websites discussing risks in a balanced way be available.

\section{PDSA 10: aim: to improve patient satisfaction with} debriefing process. Intervention: staff involvement in restructuring the debrief form

Feedback from staff throughout April guided the team in complex clinical scenarios that the form may be used. It was agreed that this form would not be a sensitive way to inform people after cases of stillbirth. They also highlighted that some doctors' views were more positive toward VBAC than others. The second draft debrief form had the sentence 'we would recommend a caesarean-section in future because ....' to give clarity to other obstetricians in future pregnancies and to allow for future audit of the reasons given when recommending caesarean-section. Staff suggested space for extra information in certain circumstances where subsequent pregnancy may be particularly risky or where sterilisation was undertaken.

Implementation of the second draft of the debrief form incurred some specific issues with the printing arrangements. This required lengthy involvement of the team and managerial staff who were able to assist and deliver the new form in late May (online supplementary file 1).

\section{RESULTS}

Debrief form use was reviewed in ten patients a week for the 4 months of intervention. In those cases where the form was used, the patient clarity survey was undertaken.

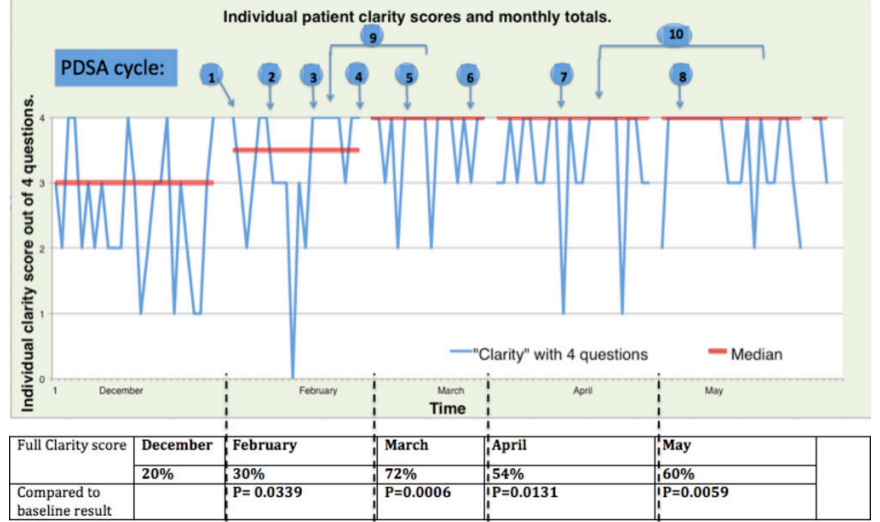

Figure 2 Run chart of outcome measure results: patient clarity with information received after caesarean-section.

Individual patient clarity scores were charted and monthly 'full clarity' was tallied (figure 2).

Analysis demonstrated a positive impact on the outcome measure; patient clarity, with the new debriefing system. 'Full clarity' scores increased from a baseline of $20 \%$ to an average of $60 \%$ by May 2017. This exceeded our goal. Comparing each monthly clarity score to the December 2016 baseline, there was a significant improvement as illustrated by a $\mathrm{p}$ value $<0.05$.

When reviewing the process measure, the use of the debrief form showed fluctuating results with increases linked to steady promotion of the project (figure 3 ). The balancing measure showed that the form required $2 \mathrm{~min}$ of operator time to fill but the overall team recouped this. Junior doctors identified time gains with potential for efficient future midwife and GP encounters.

Documented debrief in the patients notes steadily increased throughout the project. Although there was little documented evidence of verbal debriefing preceding the project, in February documented verbal debrief by operator was 33\% and this rose to $70 \%$ in May. Debrief form use in May was $80 \%$ and could also be viewed as evidence of verbal debrief. Junior doctor day 1 postnatal debrief increased from $43 \%$ in February to $70 \%$ in March after the teaching completed and reached a monthly high of $87 \%$ after the 'animal league table' intervention.

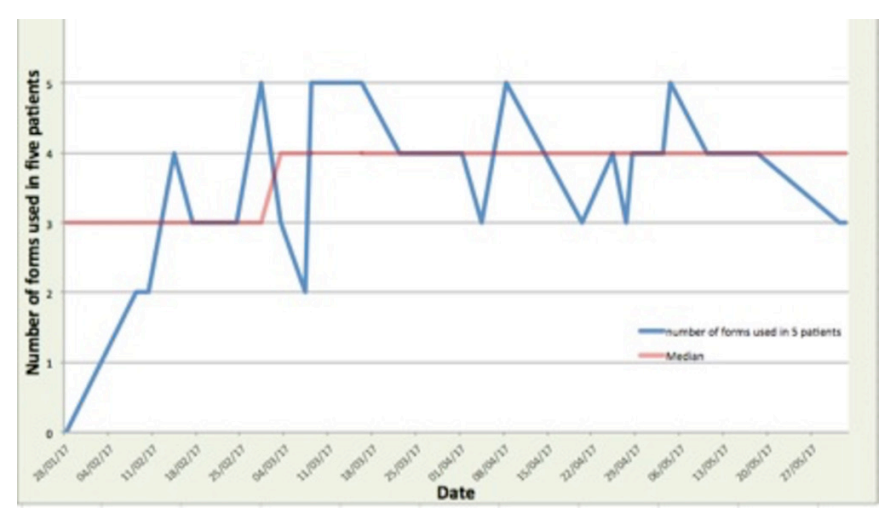

Figure 3 Run chart of process measure: number of debrief forms used (from a total of five charts per day). 


\section{LESSONS AND LIMITATIONS}

The project aimed to improve communication between obstetrician and patient after caesarean-section. One issue was the limited documentation of the debriefing process at baseline as this routinely occurred verbally. After promotion and staff education, documentation of the debriefing increased in the maternity notes but this may have occurred as frequently before intervention but without documentation. Improving patient clarity scores, however, would suggest that the project did bring communication to the fore.

A limitation of study design was obtaining clarity scores only in those who received the debrief form. We felt this was appropriate as we knew baseline data showed low clarity scores with previous methods. This collection method identified that the intervention improved this score. We understood uptake of the form would initially be challenging so wanted to quickly assess whether this intervention was worth promotion. We used these results to encourage staff buy-in to using the form. Our results however, reflect clarity scoring for perfect usage of the form which requires a longer time scale to embed into routine practice.

A lesson learnt by our team was to engage senior managerial team members early. Our failure to realise this caused delays when our redrafted form required printing and encountered problems. The 'Specialist Trainee Engaged in leadership Programme' team, provided experienced staff to educate us on these quality improvement points. This resource provided by the Belfast Trust was invaluable to the success of the project. The delay in printing the form's second draft, and its late introduction, limited comprehensive review of its success within the 6-month project timeframe. Another learning point was the importance of involving the whole healthcare team. The midwifery area leads proved invaluable in encouraging the clinical staff in each area to participate and create change.

Time limitation had its strengths and weaknesses. While weekly review prompted fast action, the speed with which interventions were introduced caused difficulty in assessment of which cycles produced the greatest benefit. Grassroot feedback suggested it was the consistent promotion of the project by multiple techniques that increased awareness and engagement of staff. Our project time period avoided the junior doctor changeover date which creates difficulties in maintaining progress in any intervention. To improve sustainability, we created a permanent staff champion to promote the project in the long-term and embed it into practice. We aim to repeat similar data collection to ensure the intervention is sustained. The 6-month period does not identify long-term advantages of the project. At a later date, when the new debrief process is embedded into practice, we aim to review how this has benefited primary care doctors and whether it impacts on women's birth choice in subsequent pregnancy. It is anticipated that further positive outcomes will be seen when women subsequently return to maternity services with a clearer understanding of previous events and will lead to informed decision making.

\section{CONCLUSION}

This project identified the information women desire after caesarean-section. With multidisciplinary support, we exceeded our aim, showing a significant improvement in information clarity among service users with little operator time expenditure. This is consistent with the previous literature that women appreciate information as soon as possible about their delivery. ${ }^{12}$ Improving consistent information through the patient journey could have far reaching implications for future deliveries. Confident affirmation that VBAC is an option for women can shape their decisions made regarding future mode of delivery. ${ }^{11}$ The small financial cost incurred is outweighed by the potential for improved efficiency in care for midwives, GP's and future antenatal clinics. Promotion of these beneficial effects should aid sustainability. Permanent staff champions will carry the project forward. Next steps include working on sustainability and promotion to primary care. Sharing this project with the greater medical community can encourage results to be replicated and lessons built on, to the advantage of many women.

Acknowledgements We thank 'Specialist Trainees Engaged in leadership Programme' (STEP) Belfast faculty who provided expert support, Dr Lucy Curran as GP liaison lead and all the staff in RJMH for cooperating with this project.

Contributors CD: designed the study, and contributed in the analysis of data. She drafted the article. ES: contributed in the collection and analysis of data. Helped with drafting the article and revised it for intellectual content. JP: contributed in the collection and analysis of data. Helped with drafting the article and revised it for intellectual content. AMN: contributed in design of the study and analysis of data. Revised the article for intellectual content. KJ: oversaw the project. Contributed in design of the study and analysis of data. Revised the article for intellectual content. All authors have approved the final draft intended for publication.

Funding The authors have not declared a specific grant for this research from any funding agency in the public, commercial or not-for-profit sectors.

\section{Competing interests None declared.}

Patient consent for publication Not required.

Provenance and peer review Not commissioned; externally peer reviewed.

Open access This is an open access article distributed in accordance with the Creative Commons Attribution Non Commercial (CC BY-NC 4.0) license, which permits others to distribute, remix, adapt, build upon this work non-commercially, and license their derivative works on different terms, provided the original work is properly cited, appropriate credit is given, any changes made indicated, and the use is non-commercial. See: http://creativecommons.org/licenses/by-nc/4.0/.

\section{REFERENCES}

1 General Medical Council. Good medical practice [Internet], 2013. Available: https://www.gmc-uk.org/guidance/good_medical_ practice/duties_of_a_doctor.asp [Accessed Mar 2018].

2 NHS Education for Scotland. Benefits of good communication. [Internet], 2017. Available: http://www.nes.scot.nhs.uk/educationand-training/by-theme-initiative/communicating,-connecting,-caring/ developing-others/first-steps/benefits-of-good-communication.aspx [Accessed Mar 2018].

3 Betran AP, Torloni MR, Zhang JJ, et al. WHO statement on caesarean section rates. World Health Organisation. BJOG 2016;123:667-70.

4 Royal College of Obstetricians and Gynaecologists. Green-top guideline No. 45: birth after previous caesarean birth. RCOG: 
London, 2015. https://www.rcog.org.uk/globalassets/documents/ guidelines/gtg_45.pdf

5 Gardner K, Henry A, Thou S, et al. Improving VBAC rates: the combined impact of two management strategies. Aust N Z J Obstet Gynaecol 2014;54:327-32.

6 NHS England. Patient and public participation in commissioning health and care: Statutory guidance for clinical commissioning groups and NHS England. [Internet], 2017. Available: https://www. england.nhs.uk/wp-content/uploads/2017/05/patient-and-publicparticipation-guidance.pdf [Accessed Mar 2018]

7 Bonzon M, Gross MM, Karch A, et al. Deciding on the mode of birth after a previous caesarean section - An online survey investigating women's preferences in Western Switzerland. Midwifery 2017;50:219-27.

8 Nair M, Soffer K, Noor N, et al. Selected maternal morbidities in women with a prior caesarean delivery planning vaginal birth or elective repeat caesarean section: a retrospective cohort analysis using data from the UK obstetric surveillance system. BMJ Open 2015;5:e007434.

9 Fenwick J, Gamble J, Hauck Y. Reframing birth: a consequence of cesarean section. J Adv Nurs 2006;56:121-30.
10 Lundgren I, Begley C, Gross MM, et al. 'Groping through the fog': a metasynthesis of women's experiences on VBAC (vaginal birth after caesarean section). BMC Pregnancy Childbirth 2012;12:85.

11 Nilsson C, Lalor J, Begley C, et al. Vaginal birth after caesarean: views of women from countries with low VBAC rates. Women Birth 2017;30:481-90.

12 Nilsson C, van Limbeek E, Vehvilainen-Julkunen K, et al. Vaginal birth after cesarean: views of women from countries with high VBAC rates. Qual Health Res 2017;27:325-40.

13 Munro S, Janssen P, Corbett K, et al. Seeking control in the midst of uncertainty: women's experiences of choosing mode of birth after caesarean. Women Birth 2017;30:129-36.

14 National Institute of Clinical Excellence(NICE). Antenatal care for uncomplicated pregnancies. Clinical guideline [CG62], 2008. Available: https://www.nice.org.uk/guidance/cg62 [Accessed Mar 2018].

15 Chen M-M, McKellar L, Pincombe J. Influences on vaginal birth after caesarean section: a qualitative study of Taiwanese women. Women Birth 2017;30:e132-9. 